\title{
A novel GFP approach for the analysis of genetic exchange in trypanosomes allowing the in situ detection of mating events
}

\author{
Lewis E. H. Bingle, ${ }^{1} \dagger$ Jane L. Eastlake, ${ }^{1}$ Mick Bailey ${ }^{2}$ \\ and Wendy C. Gibson ${ }^{1}$
}

Author for correspondence: Wendy C. Gibson. Tel: +44 117928 8249. Fax: +44 1179257374. e-mail:w.gibson@bristol.ac.uk

1 School of Biological Sciences, University of Bristol, Bristol BS8 1UG, UK

2 Department of Clinical Veterinary Science, University of Bristol, Langford, Bristol BS40 7DU, UK

\begin{abstract}
Trypanosoma brucei undergoes genetic exchange in its insect vector by an unknown mechanism. To visualize the production of hybrids in the fly, a tetracycline (Tet)-inducible expression system was adapted. One parental trypanosome clone was transfected with the gene encoding Green Fluorescent Protein (GFP) under control of the Tet repressor in trans; transfection with these constructs also introduced genes for resistance to hygromycin and phleomycin, respectively. An experimental cross with a second parental clone carrying a gene for geneticin resistance produced fluorescent hybrids with both hygromycin and geneticin resistance. These results are consistent with the meiotic segregation and reassortment of the GFP and repressor genes. Fluorescent hybrids were visible in the salivary glands of the fly, but not the midgut, confirming that genetic exchange occurs among the trypanosome life cycle stages present in (or possibly en route to) the salivary glands. In conclusion, the experimental design has successfully produced fluorescent hybrids which can be observed directly in the salivary glands of the fly, and it has been shown that the recombinant genotypes were most probably the result of meiosis.
\end{abstract}

Keywords: Trypanosoma brucei, hybrids, meiosis, Green Fluorescent Protein, tsetse fly

\section{INTRODUCTION}

Trypanosomes are parasitic protozoa, which, as part of the Euglenozoa, form an early diverging branch of the eukaryote evolutionary tree (Sogin et al., 1986; CavalierSmith, 1993). They are widespread and ubiquitous parasites of vertebrates, but the best known species are those that cause disease in humans and domestic livestock. So far genetic exchange has been demonstrated experimentally in only one species, Trypanosoma brucei (Jenni et al., 1986). However, details of the mechanism remain elusive and the frequency of genetic exchange in nature is controversial (Gibson \& Stevens, 1999). Finding out how trypanosomes achieve genetic exchange is not only important for understanding gene flow in these pathogens, but also has relevance to the study of the early evolution of eukaryotes.

\footnotetext{
†Present address: School of Biological Sciences, University of Birmingham, Edgbaston, Birmingham B15 2TT, UK.

Abbreviations: CM, Cunningham's medium; GFP, Green Fluorescent Protein; UTR, untranslated region.
}

T. brucei undergoes a complex life cycle involving both mammalian and bloodsucking insect (tsetse fly) hosts. Trypanosomes in the blood of the mammal are taken up by the fly when it feeds; inside the fly, trypanosomes first differentiate and multiply within the midgut and then migrate to the salivary glands, where they again multiply and complete their development to the mammal-infective form. Jenni et al. (1986) showed that hybrids were produced after co-transmission of two parental trypanosome clones through the tsetse fly. However, genetic exchange is not an obligatory part of the trypanosome life cycle and, although it may occur quite frequently $(30-40 \%)$ in infected flies (Schweizer et al., 1988; Turner et al., 1990), experimental flies are refractory to infection. These factors, coupled with the relative inaccessibility of genetic exchange within the tsetse fly vector, have hampered direct observation of the process. After analysis of crosses using selectable drug resistance markers, hybrids were found only in trypanosome populations derived from the salivary glands, not midguts, suggesting that genetic exchange probably takes place in the salivary glands (Gibson \& Bailey, 1994; Gibson et al., 1997b). 
Parents
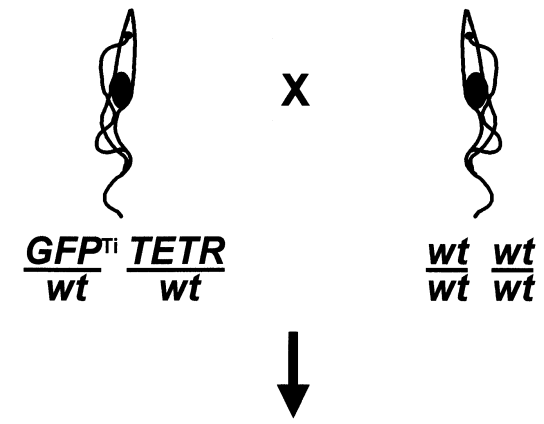

Progeny
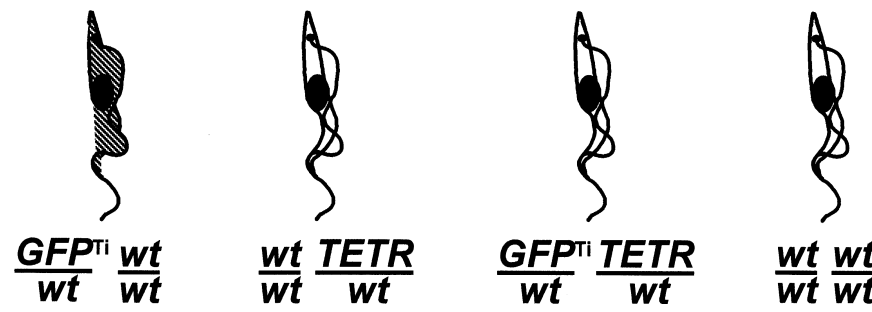

Fig. 1. Diagram illustrating the design of the experimental cross. Only the shaded hybrid progeny trypanosome will fluoresce. $G F P^{T i}$, Tet-inducible GFP gene.
Analysis of the inheritance of genetic markers in hybrid progeny suggests that a meiotic division occurs at some stage (Paindavoine et al., 1986; Sternberg et al., 1988, 1989; Gibson, 1989; Turner et al., 1990; Gibson \& Stevens, 1999), but triploid hybrids also occur with some frequency (Paindavoine et al., 1986; Wells et al., 1987; Gibson et al., 1992, 1997b; Gibson \& Bailey, 1994; Hope et al., 1999) and a haploid life cycle stage has not been found (Shapiro et al., 1984; Tait et al., 1989). The observation that kinetoplast (mitochondrial) DNA is inherited from both parents in hybrid progeny supports the surprising suggestion that somehow the complex parental kinetoplast DNA networks swap DNA (Gibson \& Garside, 1990; Turner et al., 1995; Gibson et al., 1997a). This in turn presupposes fusion of the parental mitochondria, and hence cells, during genetic exchange.

To get any further towards unravelling what happens during genetic exchange, we need to see the process within the fly. This means pinpointing the developmental stage and the region of the fly where genetic exchange takes place. To do this, we need to be able to visualize hybrids in situ and distinguish them from parental trypanosomes. The new approach described here is based on the tetracycline (Tet)-inducible expression system developed by Clayton and colleagues (Wirtz \& Clayton, 1995; Biebinger et al., 1997). The inducible expression system comprises a repressor construct targeted to the tandem array of tubulin genes in $T$. brucei, where it constitutively expresses the Tet-inducible bacterial repressor protein TetR at high level, and a reporter construct with the cognate operator sequence in its promoter. The reporter is Green Fluorescent Protein (GFP), which does not require any substrate for activity and can be visualized in live or fixed cells by fluorescence microscopy or flow cytometry. Beverley and colleagues first demonstrated the feasibility of using GFP as a marker in trypanosomatids, using both live and fixed cells of Leishmania (Ha et al., 1996) and its use in T. brucei has also been reported (e.g.
Vaidya et al., 1997; Hill et al., 1999; Marchetti et al., 2000). In an experimental cross, segregation of the chromosomes carrying the GFP and repressor genes should uncouple repression, giving rise to a proportion of hybrid progeny expressing GFP (Fig. 1). A proportion of haploid forms, should they occur, will also be fluorescent. This system should enable us to search for and detect very few hybrids against a background of non-fluorescent trypanosomes.

The reporter construct is targeted to the transcriptionally inactive spacer region of the rRNA locus in the opposite transcriptional orientation to the rRNA genes to avoid run-through transcription from the rRNA promoter (Wirtz \& Clayton, 1995). To optimize the system for both bloodstream-form and procyclic trypanosomes, Biebinger et al. (1997) tested the relative efficiency of a number of different constructs with alterations to the reporter and selectable marker genes, constitutive promoter and $3^{\prime}$ untranslated region (UTR) and found that expression could be regulated over at least two orders of magnitude using the optimized systems for either procyclic or bloodstream forms.

In this paper our goal was to determine whether this challenging new approach would produce fluorescent hybrids as predicted.

\section{METHODS}

Reporter and repressor constructs. The inducible expression construct pHD676 and the Tet repressor construct pHD449 (Biebinger et al., 1997) were both kindly supplied by C. Clayton, ZMBH, Heidelberg, Germany. An enhanced GFP coding region HindIII-BamHI cassette was amplified from plasmid pEGFP-C1 (Clontech) using primers H5-EGFP (5'ACTGAAGCTTCGCCACCATGGTGAGCAAG-3') and B3-EGFP (5'-CTGGGATCCTTACTTGTACAGCTCGTCCA- $\left.3^{\prime}\right)$. This cassette was cloned into the multiple cloning site of pHD676 to make the reporter construct pHD67E (Fig. 2). The modified repressor construct pHD449ACT (Fig. 2) was derived from pHD449 (ibid.) by swapping the aldolase 3' UTR 

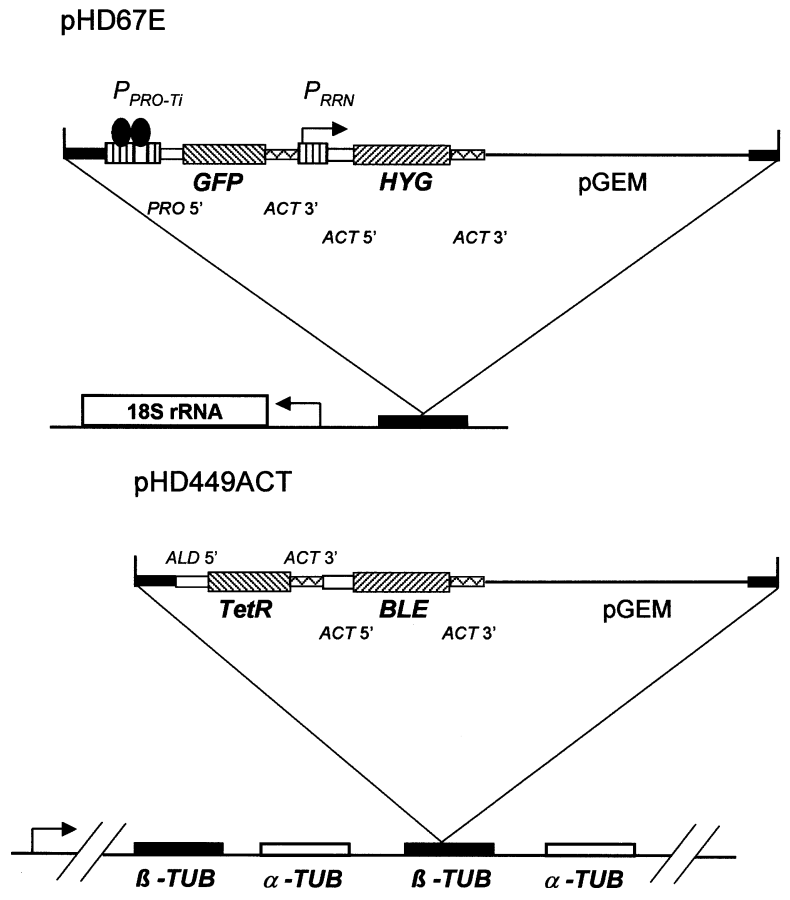

Fig. 2. Diagrams of reporter and repressor constructs. The GFP reporter construct, pHD67E, and Tet repressor construct, pHD449ACT, are shown linearized at the unique Notl site in the targeting sequences. The black boxes represent the rRNA intergenic region and $\beta$-tubulin targeting sequences, respectively. Hatched boxes represent antibiotic resistance genes (HYG, BLE) and reporter (GFP) and repressor (TetR) genes. The origins of promoter sequences, and $5^{\prime}$ and $3^{\prime}$ UTRs are indicated: $P_{P R O-T i}$ procyclin promoter with TetR binding site; $P_{R R N}$, rRNA promoter; $A C T$, actin; $A L D$, aldolase; $P R O$, procyclin. The thin line represents plasmid pGEM (Promega). Sketch maps below each construct indicate the site and orientation of expected integration into the rRNA or tubulin loci; the black box represents a non-coding region in the rRNA locus; note that the repressor construct may integrate into any $\beta$-tubulin gene in the tandem array.

cassette for the actin 3' UTR. The actin 3' UTR cassette used to make this construct was amplified from pHD30 (Hug et al., 1993) using primers ACT3BAM (5'-ACTGGATCCTAACACCGGGTTGT-3') and ACT3SMA (5'-ACGTCCCGGGAATACTGCATAGATAACAAAC-3'), which exchanged the 3' PstI site for a SmaI site. This cassette was ligated into pHD449, which had been previously digested with BamHI and partially digested with SmaI to excise the aldolase 3' UTR from the Tet $R$ coding region, while leaving the phleomycin resistance gene intact (Fig. 2). All new constructs were checked by DNA sequencing across the restriction fragment junctions involved in manipulations.

Transfection. Plasmid DNA was obtained from Escherichia coli cultures using the High Pure Plasmid Isolation kit (Boehringer Mannheim) or the Wizard PureFection DNA purification system (Promega). Mid-exponential phase procyclic trypanosomes of isolate $\mathrm{TH} 2$ [group $2 \mathrm{~T}$. b. gambiense MHOM/CI/78/TH2 (78E); Mehlitz et al., 1982], grown in Cunningham's medium (CM; Cunningham, 1977) supplemented with $10 \%$ fetal calf serum and $10 \mu$ gentamicin $\mathrm{ml}^{-1}$ ( = standard CM) at $27^{\circ} \mathrm{C}$, were transfected sequentially with reporter and repressor constructs by electroporation using two pulses of $1.5 \mathrm{kV}, 25 \mu \mathrm{F}$. Transfectants were selected $24 \mathrm{~h}$

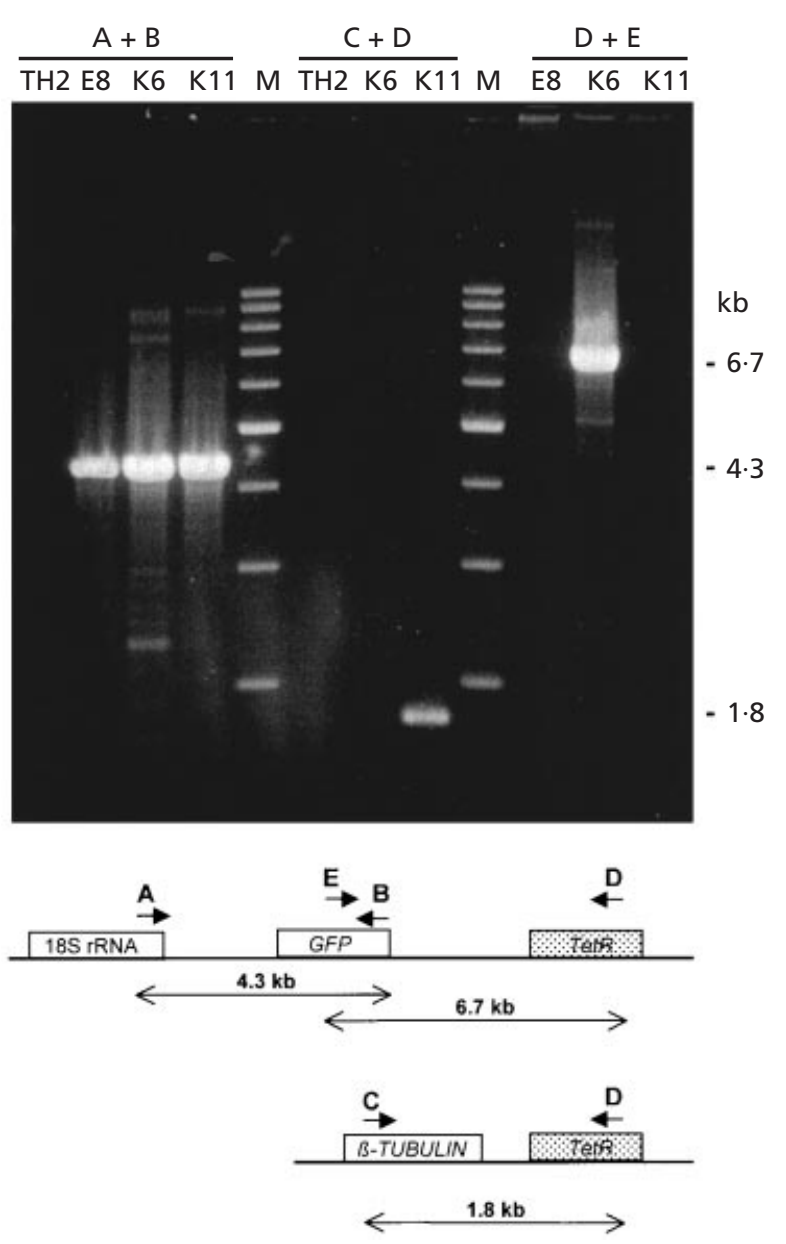

Fig. 3. $P C R$ analysis of the sites of integration of the reporter and repressor constructs into selected trypanosome clones. TH2 is wild-type; E8 is a GFP transfectant; K6 and K11 are GFPrepressed transfectants derived independently from E8. The combination of primers used for each set of PCR products is indicated and the sketch maps below show the sizes of possible amplification products, depending on the integration site of the TetR gene into the rRNA or tubulin loci. Lanes M, molecular marker $1 \mathrm{~kb}$ ladder (Promega).

post-electroporation by the addition of the appropriate antibiotic to the medium $\left(50 \mu \mathrm{g}\right.$ hygromycin $\mathrm{B} \mathrm{m}{ }^{-1} ; 1 \mu \mathrm{g}$ phleomycin $\mathrm{ml}^{-1}$ ). Clones were obtained by two rounds of limiting dilution cloning.

T. brucei genomic DNA was obtained using a simple 'miniprep' procedure (Medina-Acosta \& Cross, 1993). After digestion with appropriate restriction enzymes, DNA was fractionated by electrophoresis through $1 \%$ agarose gels. Samples for PFGE were prepared by lysing and deproteinizing trypanosomes in situ in agarose blocks (Van der Ploeg et al., 1984). Chromosomes were separated using the Pulsaphor system (Pharmacia) with a four-phase programme (Phase I, 75 min pulses at $35 \mathrm{~V}$ for $150 \mathrm{~h}$; Phase II, 20 min pulses at $60 \mathrm{~V}$ for $15 \mathrm{~h}$; Phase III, 5 min pulses at $130 \mathrm{~V}$ for $15 \mathrm{~h}$; Phase IV, $3 \mathrm{~min}$ pulses at $130 \mathrm{~V}$ for $15 \mathrm{~h}$ ) or a five-phase programme at $130 \mathrm{~V}$ (Phase I, 15 min pulses for $15 \mathrm{~h}$; Phase II, 5 min pulses for $15 \mathrm{~h}$; Phase III, $3.5 \mathrm{~min}$ pulses for $10 \mathrm{~h}$; Phase IV, $3 \mathrm{~min}$ pulses for $10 \mathrm{~h}$; Phase V, $40 \mathrm{~s}$ pulses for $10 \mathrm{~h}$ ). After acid depurination for $15 \mathrm{~min}$ in $0.25 \mathrm{M} \mathrm{HCl}$, DNA was blotted 


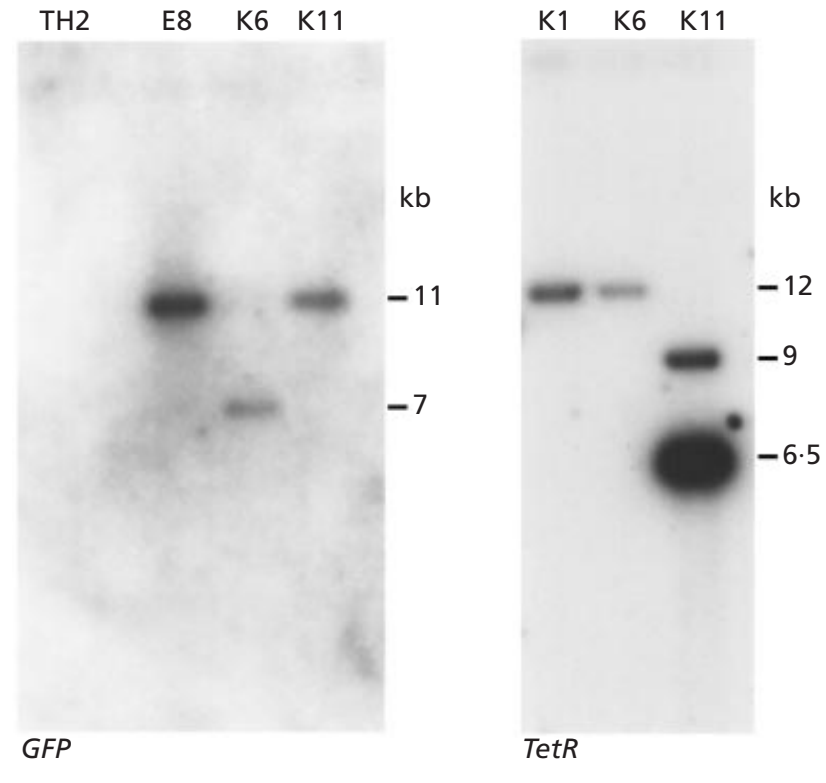

Fig. 4. RFLP analysis of sites of integration of the reporter and repressor constructs. Hindlll-digested genomic DNAs from the trypanosome clones indicated were hybridized with $\left.{ }^{32} \mathrm{P}\right] \mathrm{dCTP}-$ labelled DNA fragments as indicated. Approximate sizes are given in kb. TH2 is wild-type; E8 is a GFP transfectant; K1, K6 and $\mathrm{K} 11$ are GFP transfectants derived independently from E8.

from agarose gels onto nylon membrane (Zeta Probe; Bio$\mathrm{Rad})$ by alkaline transfer in $0.4 \mathrm{M} \mathrm{NaOH}$.

DNA probes were labelled with $\left[{ }^{32} \mathrm{P}\right] \mathrm{dCTP}$ and hybridization was carried out overnight in $0.25 \mathrm{M}$ sodium phosphate buffer $(\mathrm{pH} 7 \cdot 2), 7 \%(\mathrm{w} / \mathrm{v})$ SDS at $60{ }^{\circ} \mathrm{C}$; the membranes were then washed in $0 \cdot 1 \times$ SSC, $0.5 \%$ SDS at $60^{\circ} \mathrm{C}$.

Tsetse transmission and experimental cross. Trypanosomes were transmitted through male tsetse flies (Glossina morsitans morsitans) as described previously (Gibson, 1989). Infected flies were maintained on membrane-fed sterile horse blood supplemented with $2.5 \%(\mathrm{w} / \mathrm{v})$ bovine serum albumin (Sigma A4503) (Langley et al., 1978). Flies were dissected between 7 and 9 weeks following the infective feed. Infected midguts were cultured in CM supplemented with $10 \%$ fetal calf serum and $100 \mu \mathrm{g}$ gentamicin $\mathrm{ml}^{-1}$. Metacyclics from infected salivary glands were inoculated into mice; bloodstream forms were subsequently transformed back to procyclics if necessary, by incubation in standard CM at $27^{\circ} \mathrm{C}$.

For the experimental cross, flies were infected with a mixture of clones K11 (a GFP TetR transfectant derived from TH2) and $T$. b. bruce $i \mathrm{KP} 2 \mathrm{~N}$, a previously transfected derivative of GPAP/CI/82/KP2-I (CLONE 23) (Letch, 1984) carrying the NEO gene in the tubulin gene array (Gibson \& Whittington, 1993). Drug-resistant clones of these two parental stocks, TH2 and KP2, were successfully mated in a previous cross (Gibson et al., 1997b).

Microscopy and flow cytometry. Living trypanosomes were viewed as wet mounts in CM, blood or PBS, sometimes mounted in Vectashield (Vectalabs). Whole tsetse midguts or salivary glands were dissected into a drop of PBS and also viewed as wet mounts. Cells were generally fixed in $2 \%(\mathrm{w} / \mathrm{v})$ paraformaldehyde at $4{ }^{\circ} \mathrm{C}$ for $30 \mathrm{~min}$, if required. A DMRB microscope (Leica) equipped with either a Colour Coolview camera (Photonic Science) or Megaview II camera (Norfolk Analytical) was used for fluorescence and standard microscopy, with ImagePro Plus software (Photonic Science) or analySIS software (Norfolk Analytical), respectively.

Samples for flow cytometry were prepared by mixing a suspension of trypanosomes in PBS with an equal volume of $2 \%$ paraformaldehyde at $4{ }^{\circ} \mathrm{C}$ for $30 \mathrm{~min}$; trypanosomes were recovered by centrifugation and resuspended in PBS at approximately $10^{7} \mathrm{ml}^{-1}$. Samples were analysed using an EPICS-CS or EPICS-XL flow cytometer with Elite software (Coulter Electronics). A total of 100000 cells were collected from each sample.

\section{RESULTS AND DISCUSSION}

\section{GFP expression in procyclics}

By flow cytometry, TH2 trypanosomes transfected with the GFP construct pHD67E showed two orders of magnitude greater peak green fluorescence compared to wild-type trypanosomes, with very low numbers of weak or non-expressers $(<0.01 \%)$. The transfectants stably expressed the gene through at least 16 weeks of passage and cloning, with no apparent detrimental effects of GFP on trypanosome viability.

The correct integration of the GFP construct into the rRNA locus was confirmed by PCR across border regions and Southern analysis of restriction digests (Figs 3 and 4). The chromosomal location was determined by Southern analysis of PFGE blots (Fig. 5), since hom-

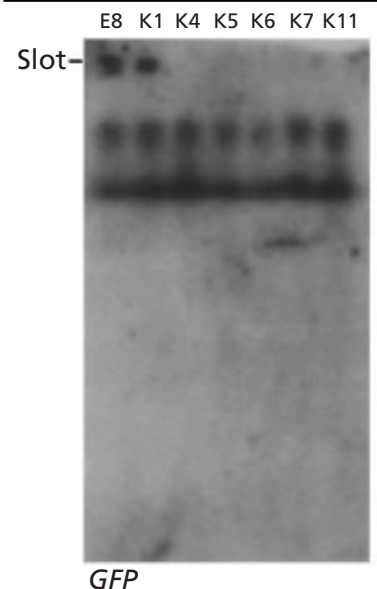

GFP
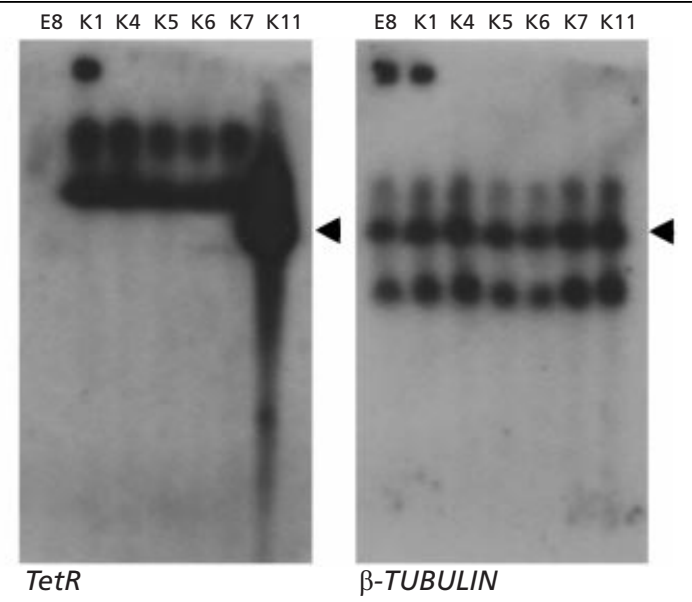

Fig. 5. Chromosomal location of sites of integration of the reporter and repressor constructs. Chromosome-sized DNA molecules from transfected trypanosome clones were separated by PFGE and probed with $\left[{ }^{32} \mathrm{P}\right] \mathrm{dCTP}$-labelled DNA fragments specific for the GFP, TetR and $\beta$-TUBULIN genes. Chromosome I, which carries the tubulin gene array, is arrowed. The hybridization signal in the slot is due to residual DNA trapped in the agarose blocks, which are still present in the $\mathrm{E} 8$ and $\mathrm{K} 1$ lanes. E8 is a GFP transfectant; K1-K11 are GFP transfectants derived independently from E8. 

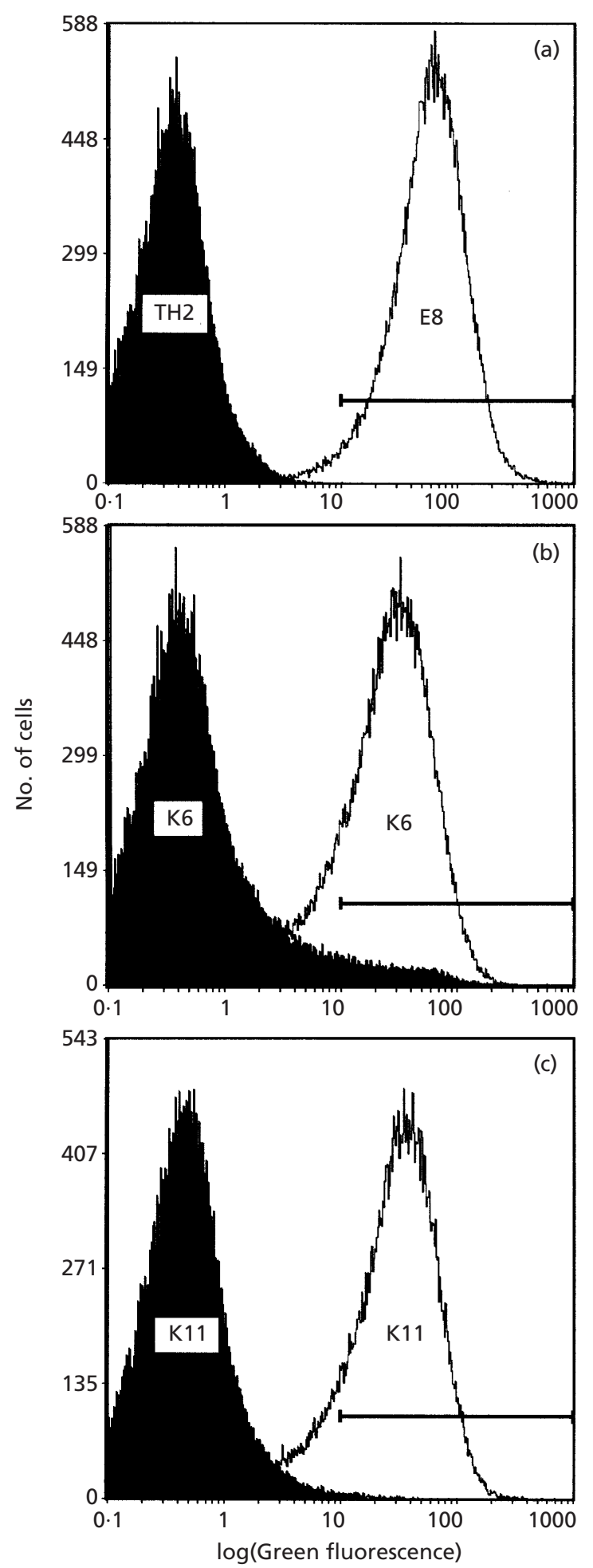

Fig. 6. Flow cytometry analysis of repression and induction of GFP expression with Tet. Each histogram represents 100000 cells. Green fluorescence was measured on a four-decade logarithmic scale in arbitrary units. The number of trypanosomes with fluorescence values within the range delineated by the horizontal bar was measured for each population. (a) Comparison of wild-type TH2 (black fill) and GFP transfectant clone E8 (no fill); (b) clone K6 ('piggyback' integration of repressor construct) grown with (no fill) and ologous chromosomes of trypanosomes often differ in size (Gibson \& Borst, 1986) and the rRNA loci are found on several pairs of chromosomes (Gottesdiener et al., 1990). The majority of transfectants were found to have the reporter construct on chromosome I, the chromosome which also carries the tubulin array (data not shown). In clone E8, however, the construct had integrated into a larger chromosome (not identified) and this clone was therefore suitable for the second round of transfection, targeting the repressor construct to the tubulin array.

\section{Control of GFP expression using the Tet repressor}

The repressor construct pHD449ACT (Fig. 2) carries a targeting sequence to direct its integration into the tubulin array of chromosome I. TH2 trypanosomes already transfected with the GFP construct and cloned (clone E8) were transfected with pHD449ACT and selected by phleomycin resistance before cloning (clones K6 and K11) and analysis. The ability of the Tet repressor to tightly regulate expression of the GFP reporter gene was assessed by examining the relative green fluorescence of cultured procyclic cells of clones K6 and K11 from uninduced (repressed) and Tetinduced (derepressed) populations. Fluorescence microscopy indicated that repression in the uninduced cells was highly efficient, with the majority of cells exhibiting no visible fluorescence and the remainder only very weakly fluorescent. Tet induction resulted in levels of green fluorescence comparable to cells without repressor. Flow cytometry confirmed these observations; the majority of repressed cells had levels of fluorescence close to the wild-type, with only a tiny proportion expressing GFP at induced levels (Fig. 6). The peak fluorescences of wild-type cells and cells carrying only the reporter construct (clone E8) differed by approximately two orders of magnitude, as did the peak fluorescences of induced and uninduced cultures of clones K6 and K11. The horizontal bar in each panel of Fig. 6 defines a range of fluorescence containing $95 \%$ of the non-regulated (E8) cell population. Only $0.79 \%$ of the repressed K11 population is within this same fluorescence range, compared with $0.06 \%$ of the wildtype (TH2) population. However, the proportion of the repressed K6 population within this range is clearly not negligible $(=5 \cdot 01 \%)$, and thus there were differences in the efficiency of repression between different transfectants.

Analysis of the site of integration of the Tet repressor construct by Southern analysis of PFGE blots revealed it to be co-localized with the GFP reporter gene in clone K6, but on a different chromosome in clone K11 (Fig. 5). Furthermore, consistent with efficient repression, the intensity of the signal with the TetR probe in K11 suggested the presence of multiple gene copies. These

without (black fill) Tet; (c) clone K11 (reporter and repressor constructs integrated at correct loci) grown with (no fill) and without (black fill) Tet. 

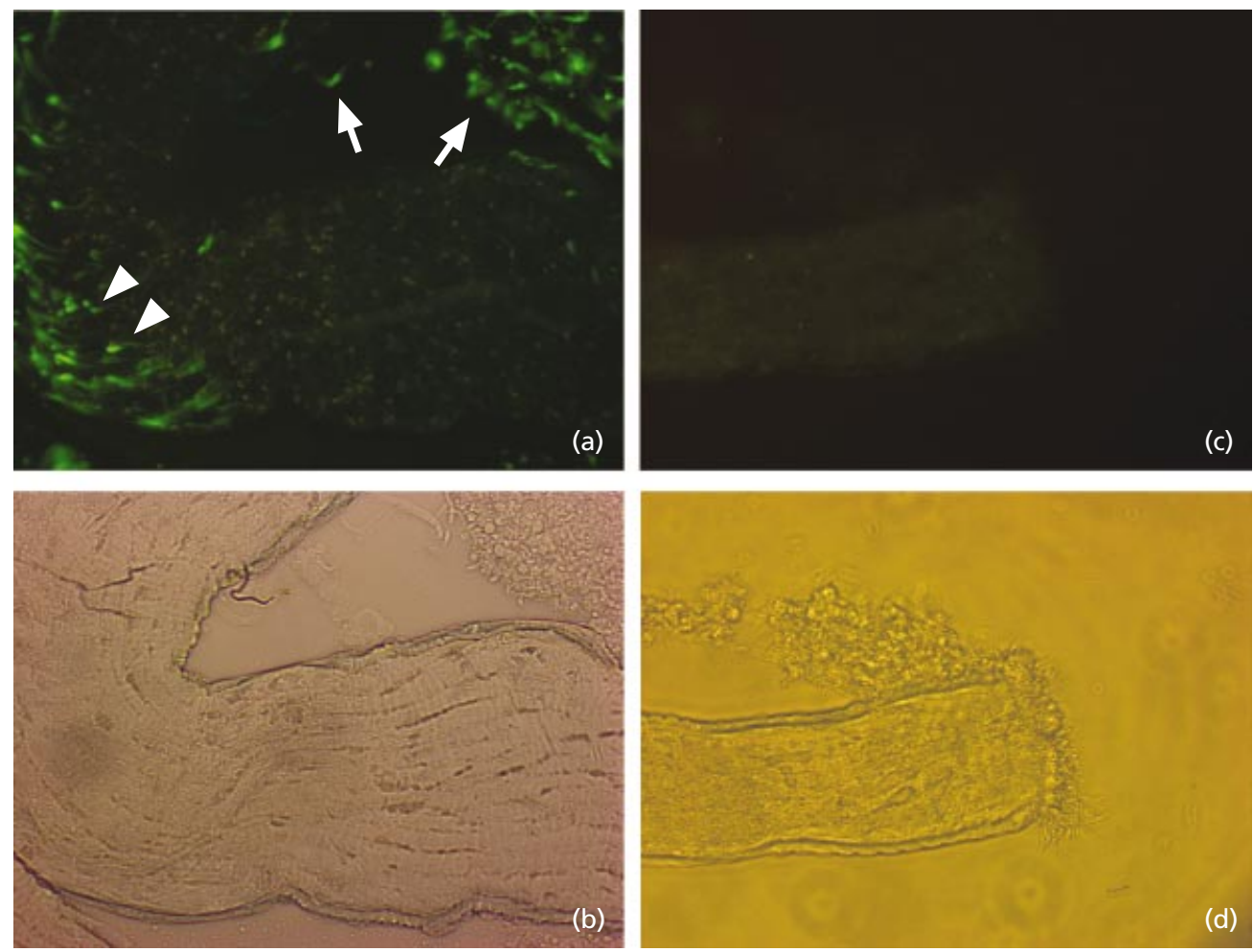

Fig. 7. Expression and repression of GFP in living epimastigotes and metacyclics within the salivary glands of the tsetse fly. (a) and (c) show images from fluorescence microscopy, while (b) and (d) are phase-contrast. (a, b) GFP transfectant E8; (c, d) GFP TetR transfectant K11. The epimastigotes (arrowheads) remain attached inside the salivary gland, while free metacyclics (arrows) can be seen outside the salivary gland at the top right (b) and spilling from the open end (d). Trypanosomes are $20-30 \mu \mathrm{m}$ in length.

results were further investigated by PCR analysis. The expected $4.3 \mathrm{~kb}$ product was produced for both clones with a GFP gene-internal primer and a primer specific for the $3^{\prime}$ end of the $18 \mathrm{~S}$ rRNA gene (Maslov et al., 1996), indicating correct integration of the reporter construct into the rRNA locus (Fig. 3). However, PCR with primers specific for the Tet repressor and $\beta$-tubulin genes, respectively, produced the expected $1.8 \mathrm{~kb}$ product only for K11, indicating integration into the tubulin locus (Fig. 3). No product was seen for clone K6; instead, a PCR product of the expected size was produced by primers specific for the GFP and TetR genes (Fig. 3), indicating that these genes are adjacent in K6. Clone K11 produced no PCR band with these primers. This suggests that in clone K6 a 'piggyback' integration of the repressor construct into the homologous plasmid DNA region of the reporter construct had occurred, with the Tet repressor gene located downstream of both the procyclin and rRNA promoters of pHD67E; the rRNA promoter would have enabled transcription of the TetR and BLE genes even if the procyclin promoter was repressed. Southern blot analysis of further clones from this transfection, and from independent transfections, suggests that a similar pattern of piggyback integration occurred frequently. This was unexpected, as the pHD449ACT construct was linearized within the $\beta$-tubulin gene targeting region prior to transfection, and this should make integration at the correct site more efficient than integration into the pGEM backbone of pHD67E (ten Asbroek et al., 1993)

Integration of the constructs was also analysed by Southern blotting of HindIII-digested genomic DNA from GFP transfectant clone E8 and the GFP-repressed transfectant clones K6 and K11 (Fig. 4). A GFP probe hybridized to fragments of approximately $11 \mathrm{~kb}$ in the $\mathrm{E} 8$ and K11 digests, consistent with integration of the pHD67E construct in the rRNA locus (Biebinger et al., 1996, 1997). In contrast, this probe hybridized to a smaller fragment (approx. $7 \mathrm{~kb}$ ) in clone $\mathrm{K} 6$. This suggests that the construct pHD449ACT, which contains a HindIII restriction site, had integrated downstream of the GFP coding region (which also has a HindIII site at the $5^{\prime}$ end) in this clone. A TetR coding region probe hybridized to different sized fragments in HindIII-digested genomic DNA from clones K1, K6 and K11 (Fig. 4), suggesting integration of pHD449 at different loci in these clones. In the K11 digest the TetR probe hybridized to fragments of two different sizes; a band of approximately $9 \mathrm{~kb}$ presumably represents a border fragment, while the much stronger hybridization to a fragment of $6.5 \mathrm{~kb}$ (approximately the same size as linearized plasmid) suggests the presence of multiple 

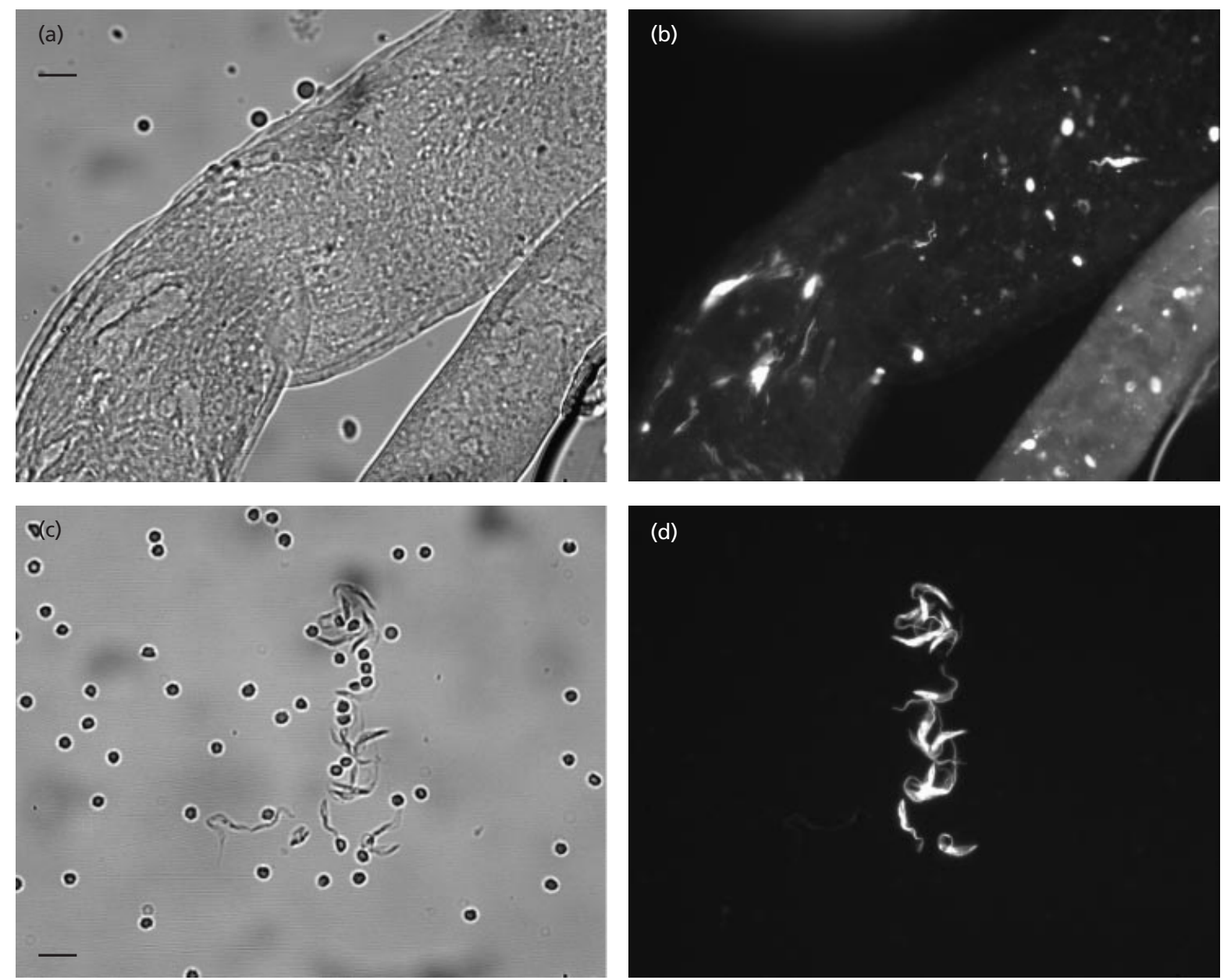

Fig. 8. Living fluorescent trypanosomes from salivary gland of fly 9. (a) Salivary gland under bright field microscopy. The salivary gland was packed full of trypanosomes, but they are not visible by bright field microscopy. (b) The same field by fluorescence microscopy; only some of the trypanosomes were fluorescent. (c, d) Transforming procyclics by light or fluorescence microscopy; some residual mouse red blood cells can be seen in (c). Two non-fluorescent trypanosomes are arrowed. Trypanosomes are 20-30 $\mu \mathrm{m}$ in length. Bars, $20 \mu \mathrm{m}$ (a); $10 \mu \mathrm{m}$ (c).

copies of the repressor construct arranged in a tandem repeat.

\section{Expression and repression of GFP through development in the tsetse fly}

The next step was to ascertain that the GFP reporter would be adequately expressed throughout the trypanosome developmental cycle in the tsetse fly and that expression would be efficiently repressed in the parental clone. The GFP transfectant clones E8 and E21 were highly visible against a background of tsetse midgut or salivary gland tissues, despite some autofluorescence of the fly tissue, and stably expressed GFP throughout cyclical development in the tsetse fly. Procyclic forms were found to express functional GFP in the relatively anoxic environment of the tsetse midgut and could be clearly visualized against the background midgut autofluorescence. Similarly, GFP expression was observed in all trypanosomes in the salivary glands, i.e. both attached epimastigote and metacyclic forms (Fig. 7). In bloodstream-form trypanosomes, however, no expression of functional GFP could be detected by fluorescence microscopy. This finding was confirmed by flow cytometry: the peak green fluorescence of bloodstream forms was just slightly greater than that of wildtype trypanosomes (not shown). When these nonfluorescent bloodstream-form trypanosomes were transformed to procyclic forms in vitro, they regained bright fluorescence. The reporter construct pHD67E was derived from pHD676 (Biebinger et al., 1997), which is designed for use in both procyclic and bloodstreamform trypanosomes; the GFP coding region is followed by $3^{\prime}$ UTR sequences from the non-developmentally regulated actin gene. The extremely low levels of GFP expression we observed may be the result of the lower activity of the procyclin promoter in bloodstream-form trypanosomes (fivefold down-regulation compared with 
Table 1. Phenotypes and PFGE karyotypes of parental clones K11 and KP2N, and 21 cloned hybrid progeny from SG9

\begin{tabular}{|lccccc|}
\hline Clone & GFP & HYG & BLE & NEO & Karyotype \\
\hline Parents & & & & & \\
K11 & - & + & + & - & TH2 \\
KP2N & - & - & - & + & KP2N \\
Progeny & & & & & \\
$1-7,9-15$ & + & + & - & - & A \\
8 & - & - & - & + & A \\
16,17, & + & + & - & + & A \\
20,21 & & & & & B \\
18 & + & + & - & - & KP2N \\
19 & - & - & - & + & \\
\hline
\end{tabular}

procyclic forms) (Biebinger et al., 1996, 1997) in combination with a reduced expression efficiency specific to GFP in this life cycle stage.

The regulation of GFP expression obtained using the Tet repressor system is clearly stable and efficient, with the vast majority of repressed cells exhibiting very little or no fluorescence. Moreover, this regulation seems to be tight throughout the life cycle in the tsetse fly, with no appreciable loss due to accumulation of mutations in the repressor/operator sequences.

\section{Experimental cross}

The GFP-repressed transfectant K11 was crossed with another trypanosome clone, $\mathrm{KP} 2 \mathrm{~N}$, carrying the $\mathrm{NEO}$ gene, conferring geneticin resistance, integrated into the tubulin locus. A mixture of fluorescent and nonfluorescent trypanosomes were observed in the salivary glands of one fly (Fig. 8a,b) and subsequently in the recovered procyclic forms (Fig. 8c,d). This population of procyclics was selected with hygromycin and geneticin separately and in combination, and 21 clones were obtained from either the drug-selected or starting populations. Of these clones, four were fluorescent and resistant to both hygromycin and geneticin, but not phleomycin. These clones were therefore unequivocal hybrids of the two parents. The phenotypes of all clones are given in Table 1. Note that few (inferred) genotypes are represented and no phleomycin-resistant trypanosomes were recovered at all; the absence of the Tet $R$ gene in these clones was confirmed by PCR using primers $\mathrm{C}$ and D (not shown). Since the original salivary gland population was expanded both in vivo and in vitro, it experienced population bottlenecks on transfer from fly to mouse and then mouse to culture, and also certain genotypes may have been favoured by growth conditions. Therefore, these clones cannot be said to represent all genotypes present among metacyclics in the salivary glands. Importantly, the trypanosomes isolated from the midgut of the same fly were not fluorescent, indicating that genetic exchange occurred in (or possibly en route to) the salivary glands.

As an independent check on their hybrid nature, clones were compared with the parents by molecular karyotype (Fig. 9). Three different karyotypes were observed among the progeny clones: two were non-parental (designated $\mathrm{A}$ and $\mathrm{B}$ ) and one was identical to that of parent KP2N. This clone (19) was not fluorescent and had geneticin resistance only, in agreement with the assumption that it represents direct transmission of $\mathrm{KP} 2 \mathrm{~N}$ without genetic exchange; genetic exchange is

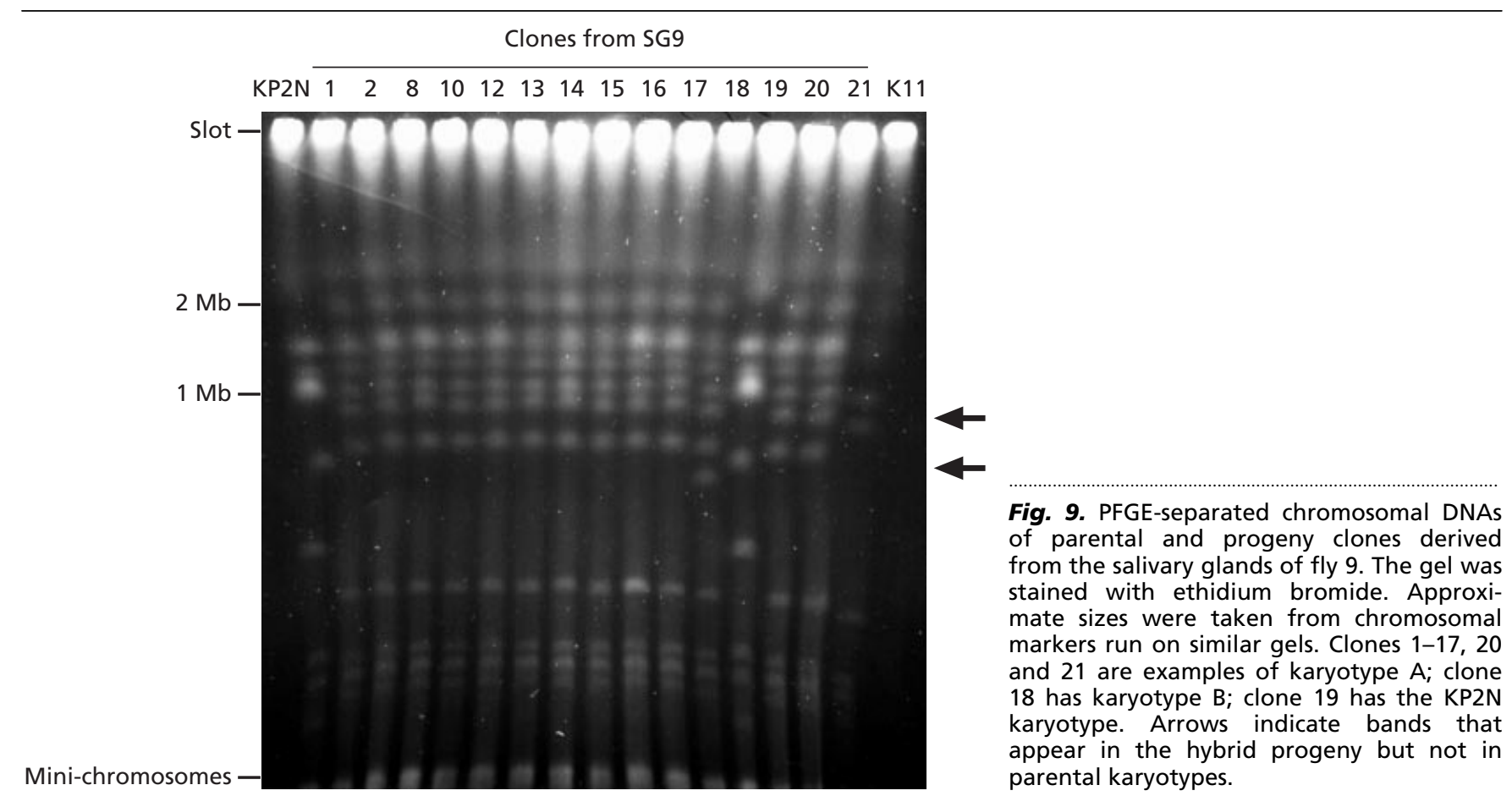


non-obligatory in T. brucei and parental genotypes are frequently recovered in the hybrid population (Schweizer et al., 1988). Karyotypes A and B appear to consist of a mixture of bands from both parents with some additional bands of non-parental sizes; the two karyotypes are similar, but karyotype $\mathrm{B}$ has one extra band relative to A. It is surprising that clones of three different phenotypes with regard to antibiotic resistance and fluorescence all shared karyotype A. One hypothesis is that clone 8 was derived from the double-drugresistant genotype shared by clones 16, 17, 20 and 21, by deletion of the reporter construct; this clone was regrown to confirm the phenotype and karyotype. Similarly, loss of the NEO gene from the double-drugresistant genotype could have produced clones 1-7 and 9-15. Alternatively, hotspots for chromosomal recombination might have generated novel non-parental chromosomes of the same size repeatedly. More detailed analysis of these clones should reveal their true relationships.

In conclusion, the experimental design has successfully produced fluorescent hybrids, which can be observed directly in the salivary glands of the fly. This opens the way to determining the life cycle stage involved in genetic exchange and a focussed search for intermediate stages. The fact that fluorescent hybrids have been produced as predicted by segregation and reassortment of alleles is further confirmation that meiosis occurs during genetic exchange.

\section{ACKNOWLEDGEMENTS}

We thank Piet Borst and Colin Lazarus for their input into the design of these experiments and Christine Clayton for generously supplying the initial constructs and sequence information. We thank the International Atomic Energy Agency, Vienna, and Mrs Jenny Berry for the experimental tsetse flies. This work was funded by grants from the UK Medical Research Council and The Wellcome Trust.

\section{REFERENCES}

ten Asbroek, A. L. M. A., Mol, C. A. A. M., Kieft, R. \& Borst, P. (1993). Stable transformation of Trypanosoma brucei. Mol Biochem Parasitol 59, 133-142.

Biebinger, S., Rettenmaier, S., Flaspohler, J., Hartmann, C., PenaDiaz, J., Wirtz, L. E., Hotz, H. R., Barry, J. D. \& Clayton, C. (1996). The PARP promoter of Trypanosoma brucei is developmentally regulated in a chromosomal context. Nucleic Acids Res 24, 1202-1211.

Biebinger, S., Wirtz, L. E., Lorenz, P. \& Clayton, C. (1997). Vectors for inducible expression of toxic gene products in bloodstream and procyclic Trypanosoma brucei. Mol Biochem Parasitol 85, 99-112.

Cavalier-Smith, T. (1993). Kingdom Protozoa and its 18 Phyla. Microbiol Rev 57, 953-994.

Cunningham, I. (1977). New culture medium for maintenance of tsetse tissues and growth of trypanosomatids. J Protozool 24, 325-329.

Gibson, W. C. (1989). Analysis of a genetic cross between Trypanosoma brucei rhodesiense and T. b. brucei. Parasitology 99, 391-402.
Gibson, W. \& Bailey, M. (1994). Genetic exchange in Trypanosoma brucei: evidence for meiosis from analysis of a cross between drug resistant transformants. Mol Biochem Parasitol 64, 241-252.

Gibson, W. C. \& Borst, P. (1986). Size-fractionation of the small chromosomes of Trypanozoon and Nannomonas trypanosomes by pulsed field gradient gel electrophoresis. Mol Biochem Parasitol 18, 127-140.

Gibson, W. \& Garside, L. (1990). Kinetoplast DNA mini-circles are inherited from both parents in genetic hybrids of Trypanosoma brucei. Mol Biochem Parasitol 42, 45-54.

Gibson, W. \& Stevens, J. (1999). Genetic exchange in the Trypanosomatidae. Adv Parasitology 43, 1-46.

Gibson, W. \& Whittington, H. (1993). Genetic exchange in Trypanosoma brucei: Selection of hybrid trypanosomes by introduction of genes conferring drug resistance. Mol Biochem Parasitol 60, 19-26.

Gibson, W., Garside, L. \& Bailey, M. (1992). Trisomy and chromosome size changes in hybrid trypanosomes from a genetic cross between Trypanosoma brucei rhodesiense and T. b. brucei. Mol Biochem Parasitol 52, 189-200.

Gibson, W., Crow, M. \& Kearns, J. (1997a). Kinetoplast DNA minicircles are inherited from both parents in genetic crosses of Trypanosoma brucei. Parasitol Res 83, 483-488.

Gibson, W., Winters, K., Mizen, G., Kearns, J. \& Bailey, M. (1997b). Intraclonal mating in Trypanosoma brucei is associated with outcrossing. Microbiology 143, 909-920.

Gottesdiener, K., Garcia-Anoveros, J., Lee, G.-S. M. \& Van der Ploeg, L. H. T. (1990). Chromosome organization of the protozoan Trypanosoma brucei. Mol Cell Biol 10, 6079-6083.

Ha, D. S., Schwarz, J. K., Turco, S. J. \& Beverley, S. M. (1996). Use of the green fluorescent protein as a marker in transfected Leishmania. Mol Biochem Parasitol 77, 57-64.

Hill, K. L., Hutchings, N. R., Russell, D. G. \& Donelson, J. E. (1999). A novel protein targeting domain directs proteins to the anterior cytoplasmic face of the flagellar pocket in African trypanosomes. J Cell Sci 112, 3091-3101.

Hope, M., MacLeod, M., Leech, V., Melville, S., Sasse, J., Tait, A. \& Turner, C. M. R. (1999). Analysis of ploidy (in megabase chromosomes) in Trypanosoma brucei after genetic exchange. Mol Biochem Parasitol 104, 1-9.

Hug, M., Carruthers, V. B., Hartmann, C., Sherman, D. S., Cross, G. A. M. \& Clayton, C. (1993). A possible role for the 3'untranslated region in developmental regulation in Trypanosoma brucei. Mol Biochem Parasitol 61, 87-96.

Jenni, L., Marti, S., Schweizer, J., Betschart, B., Lepage, R. W. F., Wells, J. M., Tait, A., Paindavoine, P., Pays, E. \& Steinert, M. (1986). Hybrid formation between African trypanosomes during cyclical transmission. Nature 322, 173-175.

Langley, P. A., Pimley, R. W., Mews, A. R. \& Flood, M. E. T. (1978). Effect of diet composition on feeding, digestion and reproduction in Glossina morsitans. J Insect Physiol 24, 233-238.

Letch, C. A. (1984). A mixed population of Trypanozoon in Glossina palpalis palpalis from Ivory Coast. Trans $R$ Soc Trop Med Hyg 78, 627-630.

Marchetti, M. A., Tschudi, C., Kwon, H., Woolin, S. L. \& Ullu, E. (2000). Import of proteins into the trypanosome nucleus and their distribution at karyokinesis. J Cell Sci 113, 899-906.

Maslov, D. A., Lukes, J., Jirku, M. \& Simpson, L. (1996). Phylogeny of trypanosomes as inferred from the small and large subunit rRNAs: implications for the evolution of parasitism in the trypanosomatid protozoa. Mol Biochem Parasitol 75, 197-205. 
Medina-Acosta, E. \& Cross, G. A. M. (1993). Rapid isolation of DNA from trypanosomatid protozoa using a simple mini-prep procedure. Mol Biochem Parasitol 59, 327-329.

Mehlitz, D., Zillmann, U., Scott, C. M. \& Godfrey, D. G. (1982). Epidemiological studies on the animal reservoir of gambiense sleeping sickness. Part III. Characterisation of Trypanozoon stocks by isoenzymes and sensitivity to human serum. Tropenmed Parasit 33, 113-118.

Paindavoine, P., Zampetti-Bosseler, F., Pays, E., Schweizer, J., Guyaux, M., Jenni, L. \& Steinert, M. (1986). Trypanosome hybrids generated in tsetse flies by nuclear fusion. EMBO J 5, 3631-3636.

Schweizer, J., Tait, A. \& Jenni, L. (1988). The timing and frequency of hybrid formation in African trypanosomes during cyclical transmission. Parasitology 75, 98-101.

Shapiro, S. Z., Naessens, J., Liesegang, B., Moloo, S. K. \& Magondu, J. (1984). Analysis by flow cytometry of DNA synthesis during the lifecycle of African trypanosomes. Acta Trop 41, 313-323.

Sogin, M. L., Elwood, H. J. \& Gunderson, J. H. (1986). Evolutionary diversity of eukaryotic small subunit rRNA genes. Proc Natl Acad Sci US A 83, 1383-1387.

Sternberg, J., Tait, A., Haley, S., Wells, J. M., Lepage, R. W. F., Schweizer, J. \& Jenni, L. (1988). Gene exchange in African trypanosomes: characterisation of a new hybrid genotype. Mol Biochem Parasitol 27, 191-200.

Sternberg, J., Turner, C. M. R., Wells, J. M., Ranford-Cartwright, L. C., Lepage, R. W. F. \& Tait, A. (1989). Gene exchange in African trypanosomes: frequency and allelic segregation. Mol Biochem Parasitol 34, 269-280.
Tait, A., Turner, C. M. R., Le Page, R. F. W. \& Wells, J. M. (1989). Genetic evidence that metacyclic forms of Trypanosoma brucei are diploid. Mol Biochem Parasitol 37, 247-256.

Turner, C. M. R., Sternberg, J., Buchanan, N., Smith, E., Hide, G. \& Tait, A. (1990). Evidence that the mechanism of gene exchange in Trypanosoma brucei involves meiosis and syngamy. Parasitology 101, 377-386.

Turner, C. M. R., Hide, G., Buchanan, N. \& Tait, A. (1995). Trypanosoma brucei-inheritance of kinetoplast DNA maxicircles in a genetic cross and their segregation during vegetative growth. Exp Parasitol 80, 234-241.

Vaidya, T., Bakhiet, M., Hill, K. L., Olsson, T., Kristensson, K. \& Donelson, J. E. (1997). The gene for a $T$ lymphocyte triggering factor from African trypanosomes. J Exp Med 186, 433-438.

Van der Ploeg, L. H. T., Schwartz, D. C., Cantor, C. R. \& Borst, P. (1984). Antigenic variation in Trypanosoma brucei analysed by electrophoretic separation of chromosome-sized DNA molecules. Cell 37, 77-84.

Wells, J. M., Prospero, T. D., Jenni, L. \& Le Page, R. W. F. (1987). DNA contents and molecular karyotypes of hybrid Trypanosoma brucei. Mol Biochem Parasitol 24, 103-116.

Wirtz, E. \& Clayton, C. (1995). Inducible gene-expression in trypanosomes mediated by a prokaryotic repressor. Science $\mathbf{2 6 8}$, 1179-1183.

Received 8 May 2001; revised 20 July 2001; accepted 9 August 2001. 